\title{
Rare GATA5 sequence variants identified in individuals with bicuspid aortic valve
}

\author{
Elizabeth M. Bonachea', Sheng-Wei Chang ${ }^{2,3}$, Gloria Zender ${ }^{2,3}$, Stephanie LaHaye ${ }^{2-4}$, Sara Fitzgerald-Butt ${ }^{1-3}$, Kim L. McBride ${ }^{1-3}$ \\ and Vidu Garg ${ }^{1-4}$
}

BACKGROUND: Bicuspid aortic valve (BAV) is the most common type of congenital heart disease (CHD) and has a proposed genetic etiology. BAV is categorized by cusp fusion, with right-left (R-L) cusp fusion being associated with additional $\mathrm{CHD}$, and right-noncoronary cusp (R-NC) fusion being associated with aortic valve dysfunction. Loss of murine Gata5, which encodes a cardiac transcription factor, results in a partially penetrant R-NC BAV, and we hypothesize that mutations in GATA5 are associated with R-NC BAV in humans.

METHODS: A cohort of 78 BAV patients (50 with isolated BAV and 28 with associated aortic coarctation) was analyzed using Sanger sequencing to identify GATA5 sequence variants. Biochemical assays were performed to identify functional deficits of identified sequence variants.

RESULTS: We identified two rare heterozygous nonsynonymous variants, p.Gln3Arg and p.Leu233Pro, for a frequency of $2.6 \%(2 / 78)$. Both individuals with nonsynonymous variants had BAV and aortic coarctation, one R-L and one R-NC subtype. Of the nonsynonymous variants, only p.GIn3Arg demonstrated decreased transcriptional activity in vitro.

CONCLUSION: Rare sequence variants in GATA5 are associated with human BAV. Our findings suggest a genotypephenotype correlation in regards to associated CHD but not cusp fusion.

C ongenital heart disease (CHD) is the most common type of birth defect, with an estimated incidence of 6-19 per 1,000 live births (1). Bicuspid aortic valve (BAV) is the most common of these congenital heart malformations with an estimated prevalence of 1-2\% in the general population (2). BAV can occur as an isolated anomaly or may be seen in association with other cardiovascular malformations, most commonly coarctation of the aorta (CoA). BAV is also associated with the development of aortic valvular calcification and aortic stenosis/regurgitation. Despite the high prevalence of BAV and the potential for morbidity associated with the condition, the mechanisms underlying both normal aortic valve development and the derangement of this process in the development of BAV remain poorly understood.
The normal aortic valve is composed of three cusps termed the right coronary, left coronary, and noncoronary cusps. BAV represents a spectrum of valve phenotypes in which two cusps are typically fused together. In the majority of BAV cases, fusion occurs between the right coronary and left coronary cusps (R-L). The next most commonly observed phenotype is fusion of right coronary and noncoronary cusps (R-NC). Fusion of the left coronary cusp and noncoronary cusp (L-NC) is quite rare. Interestingly, R-NC BAV has been associated with a greater incidence of aortic valve pathology than R-L BAV $(3,4)$. Patients with R-NC BAV are more likely to develop clinically significant aortic valve stenosis and/or regurgitation than those with R-L BAV. The R-L phenotype, on the other hand, is more likely to be associated with additional cardiac malformations such as CoA, interrupted aortic arch, and hypoplastic left heart syndrome (4). Detailed analysis of animal models of R-L and R-NC BAV were found to be associated with distinct developmental derangements, abnormal outflow tract septation, and defective outflow tract cushion development, respectively, suggesting that they represent different etiologic entities (5).

BAV is considered to have a significant genetic etiologic component on the basis of observed familial clustering (6). Huntington et al. (7) demonstrated a 9.1\% incidence of BAV in relatives of affected individuals. Linkage studies have identified disease-linked loci for BAV (8). To date, the most common reported genetic etiology of nonsyndromic BAV has been mutations in NOTCH1 by several groups (9-12). The discovery of partially penetrant R-NC BAV in mice lacking Gata5, a zinc finger transcription factor expressed in the developing heart that is important for endocardialendothelial differentiation, resulted in the recent identification of rare GATA5 sequence variants in humans with BAV, but genotype-phenotype correlations were not studied $(13,14)$. Here, we seek to investigate the role of GATA5 mutations in a well-phenotyped cohort of individuals with BAV and BAV-CoA. We hypothesize that GATA5 variants will be predominantly associated with isolated BAV and R-NC cusp fusion in humans.

\footnotetext{
Department of Pediatrics, The Ohio State University, Columbus, Ohio; ${ }^{2}$ Center for Cardiovascular and Pulmonary Research, Nationwide Children's Hospital, Columbus, Ohio; ${ }^{3}$ The Heart Center, Nationwide Children's Hospital, Columbus, Ohio; ${ }^{4}$ Department of Molecular Genetics, The Ohio State University, Columbus, Ohio. Correspondence: Vidu Garg (vidu.garg@nationwidechildrens.org)

Received 9 December 2013; accepted 7 February 2014; advance online publication 28 May 2014. doi:10.1038/pr.2014.67
} 
RESULTS

Patient Population and Identification of GATA5 Sequence

Variants

Of the 78 patients with BAV, the majority had isolated BAV

$(n=50)$ while the remaining had associated CoA (Table 1$)$. In

Table 1. Cardiac phenotype of study population

\begin{tabular}{lccc}
\hline & BAV & BAV-CoA & Overall \\
\hline R-L & $26(33 \%)$ & $20(26 \%)$ & $46(59 \%)$ \\
R-NC & $23(29.5 \%)$ & $7(9 \%)$ & $30(38.5 \%)$ \\
L-NC & $1(1 \%)$ & $1(1 \%)$ & $2(2.5 \%)$ \\
Overall & $50(64 \%)$ & $28(36 \%)$ & \\
\hline
\end{tabular}

BAV, bicuspid aortic valve (isolated); BAV-COA, bicuspid aortic valve with coarctation of the aorta; L-NC, fusion of left coronary cusp and noncoronary cusp; R-L, fusion of right coronary cusp and left coronary cusp; R-NC, fusion of right coronary cusp and noncoronary cusp. our study population, patients with BAV and CoA were more likely to have a R-L type of BAV vs. a R-NC type of BAV, while $\mathrm{R}-\mathrm{L}$ and R-NC phenotypes were equally common in isolated BAV $(P$ value $=0.036)$. Of the 78 cases, 18 were considered familial as they had additional family members with a left ventricular outflow tract malformation (five individuals had two affected family members reported). Cardiac phenotypes of affected family members are available in Supplementary Table S1 online.

We screened for mutations by DNA sequencing of the coding regions of GATA5 in the 78 individuals with BAV. Analysis of the sequencing data revealed two variants that predicted nonsynonymous amino acid substitutions, p.Gln3Arg and p.Leu233Pro (Table 2). Both variations were identified in Caucasian males. The p.Gln3Arg variation was present as a heterozygous change in one individual with BAV-CoA and

Table 2. Rare nonsynonymous sequence variants identified in patients with BAV

\begin{tabular}{|c|c|c|c|c|c|c|c|c|}
\hline $\begin{array}{l}\text { Nucleotide } \\
\text { change }\end{array}$ & $\begin{array}{l}\text { Amino acid } \\
\text { change }\end{array}$ & $\begin{array}{c}\text { BAV } \\
\text { phenotype }\end{array}$ & $\begin{array}{c}\text { Allele } \\
\text { frequency } \\
\text { in patients }\end{array}$ & $\begin{array}{c}\text { Allele } \\
\text { frequency in all } \\
\text { EVS controls }\end{array}$ & $\begin{array}{c}\text { Allele } \\
\text { frequency in EA } \\
\text { EVS controls }\end{array}$ & $\begin{array}{c}\text { Allele } \\
\text { frequency in } \\
1000 \mathrm{G} \text { controls }\end{array}$ & $\begin{array}{c}\text { PolyPhen2 } \\
\text { analysis (score) }\end{array}$ & $\begin{array}{l}\text { SIFT analysis } \\
\text { (score) }\end{array}$ \\
\hline$c .8 A>G$ & p.Gln3Arg & $\begin{array}{l}\text { BAV-CoA, } \\
\text { R-L }\end{array}$ & 0.006 & 0.003 & 0.004 & 0.004 & $\begin{array}{l}\text { Predicted } \\
\text { damaging }(0.891)\end{array}$ & $\begin{array}{l}\text { Predicted } \\
\text { damaging }(0.001)\end{array}$ \\
\hline c. $698 \mathrm{~T}>\mathrm{C}$ & p.Leu233Pro & $\begin{array}{l}\text { BAV-CoA, } \\
\text { R-NC }\end{array}$ & 0.006 & 0.001 & 0.001 & 0.003 & $\begin{array}{l}\text { Predicted } \\
\text { damaging }(0.723)\end{array}$ & $\begin{array}{l}\text { Predicted } \\
\text { damaging (0.005) }\end{array}$ \\
\hline
\end{tabular}

BAV, bicuspid aortic valve; BAV-CoA, bicuspid aortic valve with coarctation of the aorta; EA, European American; EVS, Exome Variant Server; R-L, fusion of right coronary cusp and left coronary cusp; R-NC, fusion of right coronary cusp and noncoronary cusp; 1000G, 1000 Genomes.

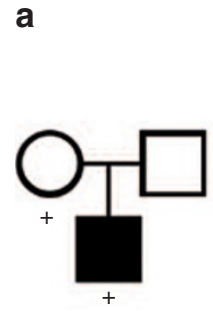

C
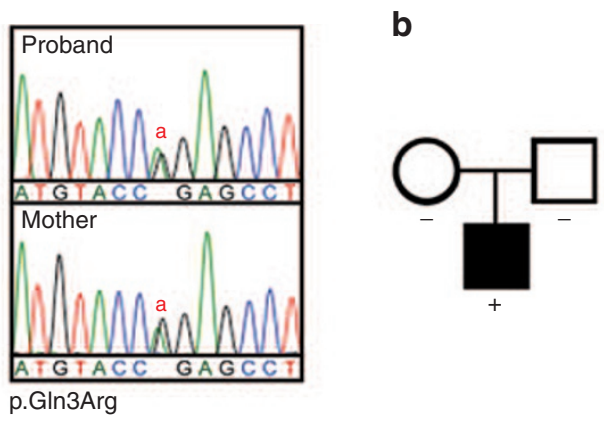

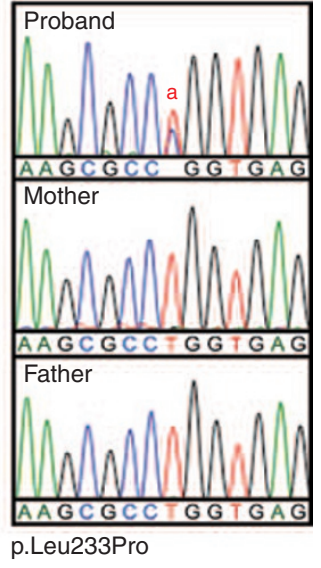

$\begin{array}{llll}10 & 229 & \text { b } & 238\end{array}$

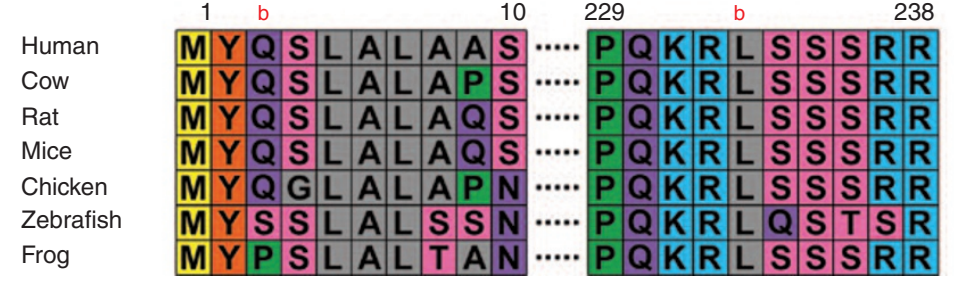

Figure 1. Rare nonsynonymous sequence variants identified in subjects with bicuspid aortic valve. (a) Family pedigree demonstrating p.GIn3Arg is inherited from unaffected mother and sequence chromatogram showing $A$ to $G$ transition which predicts the nonsynonymous amino acid substitution, p.GIn3Arg. (b) Family pedigree, which demonstrates p.Leu233Pro is a de novo mutation, and sequence chromatogram shows heterozygous T to C transition, predicting nonsynonymous amino acid substitution, p.Leu233Pro, in proband only. In pedigrees shown in (a, b), unshaded shapes indicate no known cardiac phenotype (no echocardiograms available), while shaded shapes indicate affected individuals; squares represent males while circles are females; +, presence of mutation; -, absence of mutation; ${ }^{a}$ indicates nucleotide variant position; ${ }^{b}$ indicates amino acid variant position. (c) Alignment of human GATA5 protein sequence with orthologs from multiple species. The NCBI GenBank accession numbers that were utilized for the alignment are as follows: Human-NP_536721.1,Cow-NP_001029393.1, Rat-NP_001019487.1, Mice-NP_032119.2, Chicken-NP_990752.1, Zebrafish-NP_571310.2, and Frog-NP_001081962.1. 
a

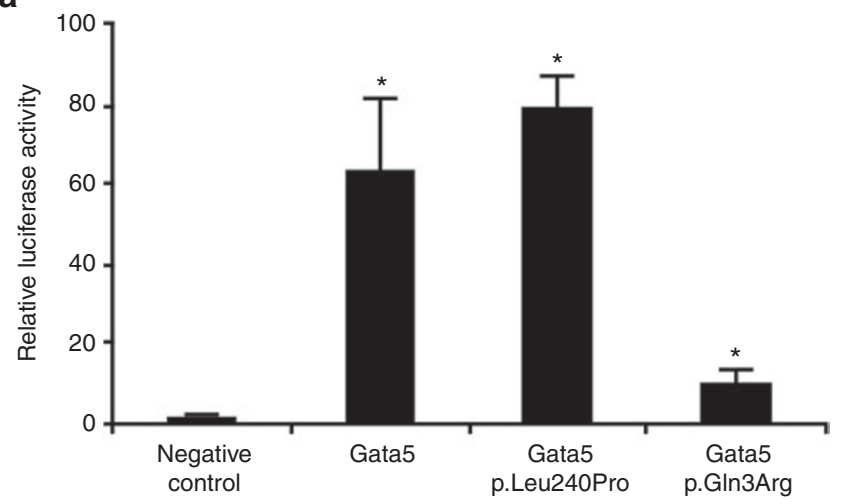

b

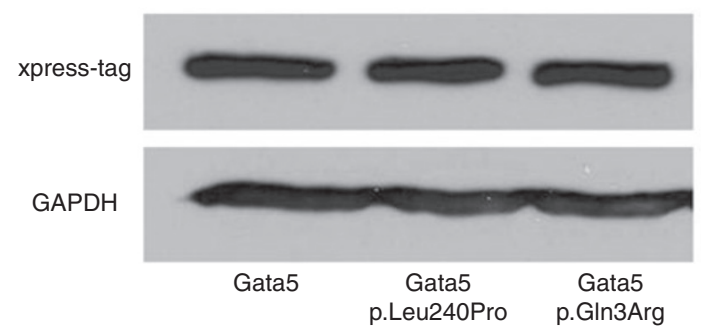

Figure 2. In vitro functional analysis of GATA5 sequence variations. (a) Transactivation assays in HeLa cells transfected with $100 \mathrm{ng}$ of murine Gata5 p.Leu240Pro and murine Gata5 p.Gln3Arg along with cotransfection of ANF-luciferase reporter and Renilla control reporter (pRL-SV40 plasmid). The $p . G \ln 3$ Arg protein demonstrated reduced activation ability as compared to wild-type Gata5 while p.Leu240Pro activation ability was unchanged. Luciferase activity is normalized to Renilla. Experiments were performed in triplicate, and means and standard deviations are shown. ${ }^{*} P<0.05$. (b) Immunoblotting demonstrates equal expression of Gata5 p.Leu240Pro and p.GIn3Arg when compared to wild type. GAPDH, glyceraldehyde 3-phosphate dehydrogenase.

$\mathrm{R}-\mathrm{L}$ cusp fusion phenotype and was inherited from the subject's mother, who is reportedly unaffected but did not undergo echocardiographic evaluation and therefore could have unrecognized BAV (Figure 1a). This individual also had a history of inguinal hernia. The mutated amino acid lies within a known transcriptional activation domain of GATA5 and is highly conserved (Figure 1c). The p.Gln3Arg variation is predicted to be damaging by in silico analysis using both PolyPhen2 and SIFT algorithms. This variation occurs at an allele frequency of 0.003 in the Exome Variant Server control population and 0.004 in the 1000 Genomes control population (Table 2).

The p.Leu233Pro variation was present as a heterozygous change in one individual with BAV-CoA and R-NC cusp fusion phenotype. The p.Leu233Pro variation was a de novo change as it was not identified in either of the proband's parents (Figure 1b). This individual also had a history of type 1 diabetes mellitus and syringomelia. Of note, there is a remote family history (maternal second cousin) of CoA. The affected amino acid occurs within the proposed nuclear localization signal and was also highly conserved (Figure 1c). The p.Leu233Pro variation is predicted damaging by in silico analysis using both PolyPhen2 and SIFT algorithms and is present at an allele frequency of 0.001 in the Exome Variant Server control population and 0.003 in the 1000 Genomes control population (Table 2).
Two rare synonymous changes at p.Ser42Ser (c.123G $>$ A) and p.Leu226Leu (c.678C $>$ T) were also identified and are detailed in Supplementary Table S2 online. The p.Ser42Ser variation was present as a heterozygous change in one Caucasian male with isolated BAV and R-NC cusp fusion phenotype. The p.Leu226Leu variation was present as a heterozygous change in one Caucasian male with isolated BAV and R-L cusp fusion phenotype. This individual has a family history of BAV in a maternal cousin (no sample available for testing). The p.Leu226Leu (c.678C $>\mathrm{T}$ ) change occurs at an allele frequency of 0.0003 in the Exome Variant Server control population, whereas the p.Ser42Ser (c.123G $>A$ ) change is not reported in this control cohort. Neither variant was reported in the 1000 Genomes control population.

\section{In Vitro Functional Analysis of Gata5 Sequence Variants}

To determine if the amino acid substitutions in GATA5 resulted in functional abnormalities, we tested murine mutant proteins analogous to the two human nonsynonymous variants found in our cohort using in vitro transfection assays. We generated mutant expression vectors in the highly conserved murine Gata5 gene for the human p.Gln3Arg and p.Leu233Pro mutations (murine p.Gln3Arg and p.Leu240Pro) and performed reporter assays using the Gata-dependent cardiac enhancer, atrial natriuretic factor (ANF), upstream of a luciferase reporter. Transfection of wild-type Gata5 demonstrated a 60-fold increase in the activation of the ANF promoter as compared to negative control (Figure 2a). While the p.Leu240Pro variant showed similar activation of the ANF-luciferase reporter as compared to wild type, the p.Gln3Arg variant had significantly decreased transactivational activity (10-fold) (Figure 2a). The Gata5 p.Leu240Pro and p.Gln3Arg mutant proteins were equally expressed in HeLa cells (Figure 2b).

\section{DISCUSSION}

Here, we identified two rare sequence variants that predict nonsynonymous amino acid substitutions in GATA5, for an estimated prevalence of $2.6 \%$ in our BAV cohort (2/78). Both nonsynonymous variants were found in individuals with BAV occurring with CoA, altered evolutionarily conserved amino acid residues, and are putatively disease causing based on in silico analysis. The p.Leu233Pro variation was a de novo change, while the p.Gln3Arg variation was identified in a presumed unaffected mother. The p.Gln3Arg mutant protein demonstrated decreased transcriptional activity in functional assays. In summary, these findings support a role for rare GATA5 sequence variants in the etiology of a minority of human BAV.

Multiple studies have established a role for rare sequence variants of cardiac transcription factors in the development of congenital heart defects in humans. Murine studies by Laforest et al. (14) suggested a role for GATA5 in human BAV when they demonstrated that Gata5-null mice had partially penetrant, R-NC BAV. Furthermore, they showed that endocardialspecific loss of Gata5 recapitulated the Gata5-null phenotype. Gata5, similar to its related transcription factors Gata4 and Gata6, is expressed in the developing heart but this expression 
is not maintained in adult cardiovascular structures, unlike Gata4 and Gata6 (ref. (15)). Murine studies demonstrated that Gata5 mRNA is present in the precardiac mesoderm and atrial and ventricular myocardium in the earliest stages of cardiac differentiation (15). At later stages of heart morphogenesis, Gata5 is predominantly expressed in the developing endocardium and endocardial cushions of the outflow tract and atrioventricular cushions, and this expression diminishes during the late stages of fetal development $(14,16)$.

A role for GATA5 in human BAV was first supported by Padang et al. (13) who identified four rare, nonsynonymous sequence variants among a cohort of 100 patients with BAV, including the p.Gln3Arg variant identified in our cohort. This study had comparable findings to our similar-sized cohort of 78 patients. Not surprisingly, Foffa et al. (9) did not identify rare, nonsynonymous GATA5 variants in their small cohort of 11 patients with familial BAV. The significance of these rare variants in disease causation remains unclear. The data supporting an etiologic link between p.Gln3Arg variants are strengthened by functional abnormalities in in vitro assays and the similar association by Padang et al. While the data supporting the p.Leu233Pro variation is less clear, its potential contribution is strengthened by its de novo inheritance, predicted functional effects by in silico analysis, and low allele frequency. As the p.Leu233Pro nonsynonymous amino acid substitution is located within the zinc finger domain, which is important for protein-protein interactions, the lack of functional effect in our in vitro transactivation assays may not be that surprising. Similar to other groups, we identified rare synonymous variants identified in our cohort $(8,13)$. Of note, the c.678C > T (Leu226Leu) variant has been previously reported in human BAV but has a low allele frequency in reference genomes (8). The possible contribution of synonymous variants to disease states such as BAV remains poorly understood. The exceedingly low allele frequencies of these changes in large control cohorts suggest a potential role in $\mathrm{BAV}$, but further investigation is required. In summary, the finding of GATA5 rare sequence variants in humans with BAV suggests that they are contributing to disease but it remains unclear if additional genetic or environmental factors are required to cause the cardiac defect.

Our studies support an etiologic role for GATA5 mutations in BAV, but genotype-phenotype correlations remain unclear. Our data are similar to Padang et al. who identified rare GATA5 sequence variants in individuals with R-L or indeterminate BAV and suggest that the cusp fusion in humans is not similar to the murine findings. Where associated cardiac defects are concerned, our data suggest a link between GATA5 and BAV occurring with CoA, whereas Padang et al. did not identify any rare, nonsynonymous variants in their subcohort of patients with BAV and coarctation. Our largely pediatric cohort limited the ability to explore genotype-phenotype correlations involving advanced valvular dysfunction and/or aortopathy. Interestingly, GATA5 variants have been reported in patients with tetralogy of Fallot, ventricular septal defects, and familial atrial fibrillation, but further studies are needed to investigate a potential role for GATA5 in isolated cases of CoA (17-19).
To date, NOTCH1 and GATA5 remain the only genes associated with isolated human BAV, but our study along with others demonstrates that variants in these genes are not involved in the majority of cases of BAV. Multiple additional candidate genes for BAV have been investigated in both human cohorts and murine models. Mutations in KCNJ2 have been linked to Andersen syndrome, which can present with BAV among other cardiovascular malformations, but no role has been identified in isolated BAV (20). Similarly, ACTA2 has been shown via linkage analysis to be associated with BAV occurring with aortic aneurysm (21). Murine models have suggested roles for Alk2, Nkx2.5, and Nos3 in BAV formation, but there have been no reported mutations of these genes in cases of human BAV (22-25). Additional investigation is required in the BAV population as there may be roles for GATA4 and GATA6, mutations in which have been associated with other types of human CHD (26-28).

In conclusion, this study supports a role for GATA5 variants in human BAV, though it remains uncertain if such variants are directly causal or serving as critical genetic modifiers. While our findings did not support the hypothesized genotype-phenotype correlation between GATA5 and cusp fusion morphology, we did find that GATA5 variants may distinguish between isolated BAV and BAV occurring with aortic coarctation, consistent with a role for GATA5 in endothelial-endocardial differentiation (29). Studies with larger populations are needed to further elucidate the relationship of GATA5 variants with both isolated $\mathrm{BAV}$ and BAV-CoA. These findings further emphasize the complex, heterogeneous genetic basis of BAV as well as the need to explore new candidate genes in large cohort studies.

\section{METHODS \\ Study Population}

The study cohort was comprised of 78 unrelated individuals (59 males and 19 females) with BAV who underwent care at Nationwide Children's Hospital. Subjects ranged in age from newborn to $38 \mathrm{y}$ old at time of enrollment $($ mean $=11 \mathrm{y})$. All subjects had clinical echocardiograms performed at Nationwide Children's Hospital with images adequate to identify cusp fusion morphology. The majority of individuals were of Caucasian ethnicity, with one African-American, one Asian, and three Hispanic individuals. Fifty of the 78 subjects (64\%) had isolated BAV, while the remainder had BAV with CoA. Fortysix subjects (59\%) had R-L cusp fusion, while 39\% had R-NC fusion and only $2 \%$ had L-NC fusion. Thirty-three subjects (42\%) had some degree of aortic stenosis at the time of enrollment; none had aortic insufficiency. The participating subjects were prospectively recruited from June 2004 to June 2011 as part of a larger study studying the genetic contributors to left ventricular outflow tract malformations. Informed consent was obtained under a protocol approved by the Institutional Review Board at Nationwide Children's Hospital; parental consent was obtained for all study participants less than $18 \mathrm{y}$ of age at enrollment with assent provided by all patients between 9-17 y old at enrollment. Medical records were reviewed to identify noncardiac congenital malformations and assess for known genetic syndromes. Subjects with known chromosomal abnormalities were excluded from the analysis. Parent samples, when possible, were also obtained under the same Institutional Review Board protocol. Genomic DNA was isolated from blood or saliva samples using the 5 PRIME DNA extraction kit (Fisher, Pittsburgh, PA).

\section{Sequencing of GATA5 and Variant Analysis}

The six exons of the coding region of GATA5 (Genbank Accession Number NM_080473) were sequenced bidirectionally via Sanger sequencing. Reference populations from the Exome Variant Server 
and 1000 Genomes Project were used as controls and therefore limited phenotype information was available in regards to subclinical cardiac anomalies $(30,31)$. When rare variants were identified in our cohort and parent DNA was available, parent samples were also sequenced. Sequencing primers are available upon request. In silico analysis was performed using the Polyphen 2 and SIFT algorithms.

\section{Plasmid Construction and Site-Directed Mutagenesis}

The mouse Gata5 expression vector was generously provided by Dr. Solway and Dr. Chen (32). The c.8A $>$ G and c.719T>C point mutations were introduced into the plasmid containing the mouse Gata5 cDNA (Genbank Accession Number NM_008093.2) to generate the mouse Gata5 p.Gln3Arg and p.Leu240Pro mutant expression vectors using the QuickChange II Site-Directed Mutagenesis Kit following the manufacturer's instructions (Agilent Technologies, Santa Clara, CA). The mutation was verified by direct sequencing. A tagged expression vector pcDNA-HisB-Gata5, which also contains an Xpress epitope tag sequence (DLYDDDDK), was also generated for the full-length murine wild type and mutant Gata5 cDNAs. These constructs were generated using PCR amplification from the original untagged expression vector using the following primers: 5'-AATTGAATTCCAAGCTCGCGCGGGGAAAA-3' and 5'-AATTCTCGAGGTGGTGACAGTTTCCTGAGC-3' (EcoRI and XhoI sites are underlined). The PCR fragments were digested with EcoRI and XhoI and cloned into the EcoRI-XhoI sites of pcDNA3.1HisB (Invitrogen, Grand Island, NY).

\section{Immunoblotting}

After transfection of wild type and mutant Gata5 tagged expression constructs into HeLa cells, $20 \mu \mathrm{g}$ of cell lysate was loaded per lane and separated using $10 \%$ sodium dodecyl sulfate-polyacrylamide gels, and transferred to immune-blot polyvinylidene difluoride membranes (Bio-rad, Hercules, CA). After blocking with 5\% nonfat milk in PBST, the membrane was probed with primary monoclonal mouse anti-Xpress (1:1,000, R910-25; Life Technologies, Grand Island, NY) and mouse anti-glyceraldehyde 3-phosphate dehydrogenase antibody (1:5,000, NB300-221; Novus Biologicals, Littleton, CO). The membrane was further probed with horseradish peroxidase-conjugated horse anti-mouse IgG (PI-2000).

\section{Transactivation Assay}

The luciferase reporter studies were performed as described previously (26). Briefly, HeLa cells were transiently transfected with 100-ng ANF-luciferase reporter plus $50 \mathrm{ng}$ pRL-SV40 plasmid, in combination with $100 \mathrm{ng}$ of wild-type Gata5, Gata5 p.Gln3Arg, or Gata5 p.Leu240Pro plasmid using Lipofectamine 2000 (Invitrogen) according to the manufacturer's protocol. Luciferase activity was measured $48 \mathrm{~h}$ after transfection using the Dual-Glo Luciferase Assay (Promega, Madison, WI) according to the manufacturer's protocol. Mean luciferase activity was calculated after normalization to Renilla. Three independent experiments were performed in triplicate.

\section{SUPPLEMENTARY MATERIAL}

Supplementary material is linked to the online version of the paper at http://www.nature.com/pr

\section{ACKNOWLEDGMENTS}

We thank the participants and their families for their involvement in this study. The authors thank the NHLBI GO Exome Sequencing Project and its ongoing studies, which produced and provided exome variant calls for comparison: the Lung GO Sequencing Project (HL-102923), the WHI Sequencing Project (HL-102924), the Broad GO Sequencing Project (HL-102925), the Seattle GO Sequencing Project (HL-102926), and the Heart GO Sequencing Project (HL-103010).

\section{STATEMENT OF FINANCIAL SUPPORT}

This work was supported by funding from the National Institutes of Health/ National Heart, Lung, and Blood Institute (R01HL088965 and R01HL109758 to V.G. and R01HL109758, R01HL090506, and R21HL106549 to K.L.M.) and The Research Institute at Nationwide Children's Hospital to V.G.

Disclosure: The authors declare no conflicts of interest.

\section{REFERENCES}

1. Hoffman JI, Kaplan S. The incidence of congenital heart disease. J Am Coll Cardiol 2002;39:1890-900.

2. Ward C. Clinical significance of the bicuspid aortic valve. Heart 2000;83:81-5.

3. Cripe L, Andelfinger G, Martin LJ, Shooner K, Benson DW. Bicuspid aortic valve is heritable. J Am Coll Cardiol 2004;44:138-43.

4. Fernandes SM, Sanders SP, Khairy P, et al. Morphology of bicuspid aortic valve in children and adolescents. J Am Coll Cardiol 2004;44:1648-51.

5. Fernández B, Durán AC, Fernández-Gallego T, et al. Bicuspid aortic valves with different spatial orientations of the leaflets are distinct etiological entities. J Am Coll Cardiol 2009;54:2312-8.

6. Emanuel R, Withers R, O'Brien K, Ross P, Feizi O. Congenitally bicuspid aortic valves. Clinicogenetic study of 41 families. Br Heart J 1978;40:14027.

7. Huntington K, Hunter AG, Chan KL. A prospective study to assess the frequency of familial clustering of congenital bicuspid aortic valve. J Am Coll Cardiol 1997;30:1809-12.

8. Martin LJ, Ramachandran V, Cripe LH, et al. Evidence in favor of linkage to human chromosomal regions $18 \mathrm{q}, 5 \mathrm{q}$ and $13 \mathrm{q}$ for bicuspid aortic valve and associated cardiovascular malformations. Hum Genet 2007;121:27584.

9. Foffa I, Ait Alì L, Panesi P, et al. Sequencing of NOTCH1, GATA5, TGFBR1 and TGFBR2 genes in familial cases of bicuspid aortic valve. BMC Med Genet 2013;14:44.

10. Garg V, Muth AN, Ransom JF, et al. Mutations in NOTCH1 cause aortic valve disease. Nature 2005;437:270-4.

11. McBride KL, Riley MF, Zender GA, et al. NOTCH1 mutations in individuals with left ventricular outflow tract malformations reduce ligand-induced signaling. Hum Mol Genet 2008;17:2886-93.

12. Mohamed SA, Aherrahrou Z, Liptau H, et al. Novel missense mutations (p.T596M and p.P1797H) in NOTCH1 in patients with bicuspid aortic valve. Biochem Biophys Res Commun 2006;345:1460-5.

13. Padang R, Bagnall RD, Richmond DR, Bannon PG, Semsarian C. Rare non-synonymous variations in the transcriptional activation domains of GATA5 in bicuspid aortic valve disease. J Mol Cell Cardiol 2012;53:27781.

14. Laforest B, Andelfinger G, Nemer M. Loss of Gata5 in mice leads to bicuspid aortic valve. J Clin Invest 2011;121:2876-87.

15. Molkentin JD. The zinc finger-containing transcription factors GATA-4, -5 , and -6 . Ubiquitously expressed regulators of tissue-specific gene expression. J Biol Chem 2000;275:38949-52.

16. Morrisey EE, Ip HS, Tang Z, Lu MM, Parmacek MS. GATA-5: a transcriptional activator expressed in a novel temporally and spatiallyrestricted pattern during embryonic development. Dev Biol 1997;183: 21-36.

17. Gu JY, Xu JH, Yu H, Yang YQ. Novel GATA5 loss-of-function mutations underlie familial atrial fibrillation. Clinics (Sao Paulo) 2012;67: 1393-9.

18. Wei D, Bao H, Liu XY, et al. GATA5 loss-of-function mutations underlie tetralogy of fallot. Int J Med Sci 2013;10:34-42.

19. Wei D, Bao H, Zhou N, Zheng GF, Liu XY, Yang YQ. GATA5 loss-of-function mutation responsible for the congenital ventriculoseptal defect. Pediatr Cardiol 2013;34:504-11.

20. Andelfinger G, Tapper AR, Welch RC, Vanoye CG, George AL Jr, Benson DW. KCNJ2 mutation results in Andersen syndrome with sex-specific cardiac and skeletal muscle phenotypes. Am J Hum Genet 2002;71: 663-8.

21. Guo DC, Pannu H, Tran-Fadulu V, et al. Mutations in smooth muscle alpha-actin (ACTA2) lead to thoracic aortic aneurysms and dissections. Nat Genet 2007;39:1488-93.

22. Biben C, Weber R, Kesteven S, et al. Cardiac septal and valvular dysmorphogenesis in mice heterozygous for mutations in the homeobox gene Nkx2-5. Circ Res 2000;87:888-95.

23. Lee TC, Zhao YD, Courtman DW, Stewart DJ. Abnormal aortic valve development in mice lacking endothelial nitric oxide synthase. Circulation 2000;101:2345-8. 


\section{Articles $\mid$ Bonachea et al.}

24. Majumdar R, Yagubyan M, Sarkar G, Bolander ME, Sundt TM 3rd. Bicuspid aortic valve and ascending aortic aneurysm are not associated with germline or somatic homeobox NKX2-5 gene polymorphism in 19 patients. J Thorac Cardiovasc Surg 2006;131:1301-5.

25. Thomas PS, Sridurongrit S, Ruiz-Lozano P, Kaartinen V. Deficient signaling via Alk2 (Acvr1) leads to bicuspid aortic valve development. PLoS One 2012;7:e35539.

26. Maitra M, Koenig SN, Srivastava D, Garg V. Identification of GATA6 sequence variants in patients with congenital heart defects. Pediatr Res 2010;68:281-5.

27. Schluterman MK, Krysiak AE, Kathiriya IS, et al. Screening and biochemical analysis of GATA4 sequence variations identified in patients with congenital heart disease. Am J Med Genet A 2007;143A:817-23.
28. Tomita-Mitchell A, Maslen CL, Morris CD, Garg V, Goldmuntz E. GATA4 sequence variants in patients with congenital heart disease. J Med Genet 2007;44:779-83.

29. Nemer G, Nemer M. Cooperative interaction between GATA5 and NFATc regulates endothelial-endocardial differentiation of cardiogenic cells. Development 2002;129:4045-55.

30. Exome Variant Server, NHLBIGO Exome Sequencing Project(ESP). Seattle, WA http://evs.gs.washington.edu/EVS/.

31. Abecasis GR, Auton A, Brooks LD, et al. An integrated map of genetic variation from 1,092 human genomes. Nature 2012;491:56-65.

32. Chen B, Yates E, Huang Y, et al. Alternative promoter and GATA5 transcripts in mouse. Am J Physiol Gastrointest Liver Physiol 2009;297: G1214-22. 